\title{
AN OBJECTIVE EXPLICIT FORMULATION BASED ON THE OLDROYD-B CONSTITUTIVE EQUATION
}

\author{
G. ${ }^{\mathrm{a}}$ Mompean \\ R. L. ' Thompson \\ P. R. ${ }^{\text {b Souza Mendes }}$ \\ a) Laboratoire de Mécanique de Lille, \\ Université des Sciences et \\ Technologies de Lille, EUDIL. \\ Cité Scientifique, 59655. Villeneuve d'Ascq \\ Cedex, France. \\ b) Department Mechanical Engineering, \\ Pontifícia Universidade Católica \\ Rio de Janeiro. \\ Rua Marquês de São Vicente, 225. Gávea, \\ Rio de Janeiro, 22453-900, Brazil

\begin{abstract}
In the present paper is shown how to obtain an objective explicit algebraic extra-stress model (AESM) based on differential constitutive equations for viscoelastic flows (Oldroyd B, White-Metzner, Phan-Thien-Tanner, etc). The formulation is developed for three-dimensional time-dependent flows. In a previous work, Mompean, 1998 obtained a non-objective algebraic model. This inconsistency is now removed introducing the relative-rate-ofrotation tensor making the model frame-invariant. A new generalized objective time derivative is also introduced, giving flexibility to the model. Calculations are performed with an Oldroyd B based AESM for a 4:1 contraction flow, showing good agreement with the original constitutive differential model. Several results with particular derivatives are obtained and an analysis guided by a flow type classifier is given. This formulation is able to include a prediction, not present in the Oldroyd B model, the viscometric second normal stress difference, $N_{2}$.
\end{abstract} \\ Keywords: non-Newtonian fluids, mathematical model
}

\section{INTRODUCTION}

Numerical prediction of viscoelastic fluids flowing through three-dimensional (3D) complex geometries is an area of great interest, encompassing industrial and scientific applications.

Viscoelastic liquids are commonly found in the industries of plastics, food, paints, petroleum, to name a few. In these industrial applications, viscoelastic liquids not rarely flow through complex three-dimensional geometric configurations, and their viscoelastic nature often requires multi-mode constitutive models to truthfully represent their mechanical behavior. Examples where timedependent effects are present also abound in industrial processes.

A great challenge while performing numerical simulations of these flows is to solve the mass and momentum conservation equations in conjunction with a constitutive equation for stress. The resulting

system of equations is a mathematical description of a physical problem which involves complex phenomena and effects, such as elasticity, inertia and diffusion. For example, in the case of a three-dimensional flow of a viscoelastic fluid described by any differential single-mode constitutive model, the task is to solve ten coupled differential equations (namely, six for the extra-stress tensor, three for momentum conservation, and one for mass conservation).

This number can increase rapidly for multimode fluid models (Baaijens, 1993\}. The computing time and the storage memory are important parameters that can reach huge values for 3D numerical simulation of multimode viscoelastic fluids. Even with the use of powerful modern computers and the most efficient algorithms, performing such simulations still constitutes a formidable task. Due to this reason, the quest for simplified viscoelastic models for numerical simulations has become an important area of research in the last years. The use of the algebraic extrastress model (AESM) formulation (Mompean, 1998) goes in this direction.

The aim of the present study is to propose a method for obtaining objective explicit algebraic extra-stress models starting from any differential constitutive equation, such as, for example, the Upper-Convected Maxwell, OldroydB, White-Metzner and Phan-Thien-Tanner models. The procedure is based on the transformation of the constitutive equation into an algebraic objective model, while keeping its elasticity prediction capability. One differential equation only has to be solved, namely, for the trace of the extra-stress tensor.

Inspired in the analogy between viscoelastic fluids and turbulence _odeling mentioned by Rivlin, 1957, this approach was recently applied to flows of viscoelastic liquids by Mompean, 1998 and Mompean et al., 1998, to obtain constitutive models via polynomial base expansions (Pope 1975). The approach is interesting because it keeps the capability of predicting viscoelasticity effects. However, the models proposed in these recent works are not objective, because the vorticity tensor $\boldsymbol{W}$ has been used as a base to develop the polynomial expansion.

Objectivity is attained in the present work by modifying a previously used hypothesis for the advection of extra-stress. In this connection, two new kinematic tensors (see Astarita 1979) are needed, namely (i) $\Omega$, the rate of rotation of the principal directions of $S$, the rate-ofdeformation tensor and (ii) $\overline{\boldsymbol{W}}=\boldsymbol{W}-\boldsymbol{\Omega}$. The relativerate-of-rotation tensor, $\overline{\boldsymbol{W}}$, is objective and measures the rate of rotation of a material particle as seen by an observer which is fixed to the principal axes of $\boldsymbol{S}$. 
Inspired in the early work by Schunk and Scriven, 1990, Souza Mendes et al., 1995, and later Thompson et al., 1999 employed $\overline{\boldsymbol{W}}$ to produce algebraic constitutive models which predict rheological material functions for stationary flow, but are not capable of predicting transient elasticity effects.

Flows of non-Newtonian viscoelastic fluids inside or around complex geometries having singularities also rather difficult to simulate numerically. For this reason, the flow through abrupt planar or axisymmetric contractions is often employed as a test flow for numerical simulations in the field of non-Newtonian fluid mechanics, and will also be used here to study the present formulation. Even for the Newtonian case, the flow originated by this geometry is complex in a sense that the fluid is submitted to a mix of shear, extension and solid body motion. The singularity present in the sharp corner of this geometry is responsible for many difficulties that arise in numerical simulations of viscoelastic flows. The strong gradient near the singularity for pressure and extra-stress components can introduce numerical errors.

In the present study we present solutions for the 2D flow through a planar contraction, using the model described later in this paper. The paper is organized as follows. In Section 2 the conservation equations are presented. The objective algebraic extra-stress model strategy is introduced in Section 3 and applied to the Oldroyd-B model. The finite volume numerical method used to discretize the equations is discussed in Section 4. Numerical results for the flow through the 4:1 contraction are presented in Section 5, together with a discussion of some important features of the proposed model. Finally, the conclusions are given in Section 6.

\section{GOVERNING EQUATIONS}

The mass and momentum conservation equations coupled with the constitutive relation for the extra-stress components of an Oldroyd-B fluid are considered in this section. The velocity field $\boldsymbol{v}$ as well as the independent variables are non-dimensionalised using a characteristic velocity scale $U$ and length scale $L$ are taken as the average velocity in the downstream half channel and the width of the downstream half channel $(H)$, respectively (see Fig. 1). The pressure $(p)$, and extra-stress $(\tau)$ variables are scaled with $\eta U / L$. The viscosity $\eta$ will be equal to the sum of the Newtonian viscosity $\eta_{0}$ and the polymeric viscosity $\eta_{1}$, then the Reynolds number is defined as $\operatorname{Re}=\{\rho U L\} / \eta$, where $\rho$ is the density. The viscosities are non-dimensionalised as $\eta_{i}=$ $\eta_{i} / \eta, I=0,1$. The Deborah number is given by $D e=\lambda \dot{\gamma}$, where $\lambda$ is the relaxation time of the viscoelastic fluid, and $\dot{\gamma}=U / L$ is the characteristic shear rate in the downstream channel for the contraction flow. mass conservation:

$$
\nabla \cdot \boldsymbol{v}=0
$$

(ii) momentum conservation:

$$
\operatorname{Re} \frac{D v}{D t}=\nabla \cdot(-p I+2 \eta o S+\tau)
$$

(iii) Oldroyd-B constitutive equation:

$$
\tau+D e\left(\frac{D \tau}{D t}-\nabla v^{T} \tau-\tau \nabla v\right)=2 \eta_{1} S
$$

where $D / D t$ is the material derivative, $\boldsymbol{I}$ the identity tensor, and $\boldsymbol{S}$ the (symmetric) rate of deformation tensor. The deformation rate tensor is defined as:

$$
S=\frac{1}{2}\left(\nabla v+\nabla^{T} v\right)
$$

where the following definition has been used for the velocity gradient $(\nabla v)_{i j}=\frac{\partial u_{j}}{\partial x_{i}}$ and $\nabla^{T} v$ is the transpose.

\section{OBJECTIVE ALGEBRAIC EXTRA-STRESS FORMULATION}

This section is divided in three sub-sections. In the first one, the general equations and the hypothesis to obtain the algebraic extra-stress model is presented and applied to the Oldroyd-B model. In the second section, a way of including the second normal stress difference for pure shear flows, $N_{2}$ to the model is shown. In the third, a classifier sensitive to the type of the flow (shear, extension and solid body motion) is analyzed.

\section{General Equations}

The constitutive equation Eq. (3) for the viscoelastic model can be rewritten in terms of the kinematic tensors $\boldsymbol{S}$ and $\boldsymbol{W}$, where $\boldsymbol{W}$ is the vorticity tensor (skew-symmetric) defined as:

$$
W=\frac{1}{2}\left(\nabla v-\nabla v^{T}\right)
$$

It is convenient to put in this form in order to work with the tensor polynomial expansion basis. This is done straightforward by replacing the velocity gradient by $\nabla v=S+W$, and its transpose by $\nabla v^{T}=S-W$. The equation Eq. (3) is now rewritten using $\boldsymbol{S}$ and $\boldsymbol{W}$ as:

$$
\frac{D \tau}{D t}=-\frac{1}{D e} \tau+\frac{2 \eta 1}{D e} S+(S \tau+\tau S)-(W \tau-\tau W) .
$$

In order to develop an explicit algebraic extra-stress model using the kinematic tensors $\boldsymbol{S}$ and $\boldsymbol{W}$, (Rivlin and Ericksen, 1955) have shown that a linear relation can be obtained between a depend tensor and a finite number of others tensors (basis tensors) formed from the elements of the kinematic tensors $\boldsymbol{S}$ and $\boldsymbol{W}$ (the independent tensors). Following (Rivlin and Ericksen, 1955), we have to work with a traceless matrix, in this way the extra-stress traceless tensor $\boldsymbol{\Gamma}$ is introduced and defined as: 


$$
\Gamma=\tau-\frac{I_{\tau}}{3} I
$$

where $I_{\tau}=\{\tau\}$ is the first invariant (the trace) of the tensor $\boldsymbol{\tau}$. Then Eq. (6) can be expressed in terms of $\boldsymbol{\Gamma}$ as:

$$
\frac{D \Gamma}{D t}=-\frac{1}{D e} \Gamma+\left(S \Gamma+\Gamma S-\frac{2}{3}\{\Gamma S\} I\right)-(W \Gamma-\Gamma W)+2\left(\frac{\eta_{1}}{D e}+\frac{1}{3} I_{\tau}\right) S .
$$

In order to close Eq.(8), an evolution equation for $\mathrm{I}_{\tau}$ is needed, which can be obtained directly from Eq. (6). Taking the trace of Eq. (6) and multiplying the Eq. (7) by the deformation rate tensor $S$ and taking its trace we can write after some algebraic manipulation:

$$
\frac{D I_{\tau}}{D t}=-\frac{1}{D e} I_{\tau}+2\{\Gamma S\}
$$

In order to apply the strategy, using a polynomial expansion, to obtain an explicit relation for the viscoelastic extra-stress tensor, it is necessary to assume an algebraic form for $\frac{D \Gamma}{D t}$, on the left hand side of Eq. (8). This is done by requiring that the extra-stress anisotropy tensor $b=\frac{\Gamma}{I_{\tau}}$ of a particle is constant, reaching an equilibrium state:

$$
\frac{D^{\circ} \Gamma}{D t} \approx \frac{\Gamma D}{I_{\tau}} \frac{I_{\tau}}{D t}
$$

This algebraic assumption is a consistent representation for two- and three-dimensional flows. For the case of viscometric flows, it is exactly verified (for details see Mompean, 1998). Besides that in nearly viscometric flows, where local effects dominate transport effects, this approach may provide an adequate representation of the extra-stresses. The other thing to notice is that using this assumption the transport terms of the extra-stresses are not completely neglected but replaced by the transport terms of the trace $\mathrm{I}_{\tau}$.

In order to be consistent to the principle of the frameindifference of the constitutive operator, the time derivative that appears on the left side of Eq.(10) can not be the material derivative. (Thompson, 2001), has proposed a new general form of an objective time derivative for a tensor of second order $M$ :

$$
\frac{D^{\circ} M}{D t}=\frac{D M}{D t}-c_{1}(S M+M S)-c_{2}(M W-W M)-\left(1-c_{2}\right)(M \Omega-\Omega M),
$$

where $c_{1}$ and $c_{2}$ are constants and $\boldsymbol{\Omega}$ is a tensor related to the rate of rotation of the principal directions of $\boldsymbol{S}$. This time derivative satisfies invariance (the operator is the same for all observers in relative motion) and preserves symmetry (in the sense that this rate of a symmetric tensor is necessarily symmetric) for all values of $c_{1}$ and $c_{2}$.
Particular choices of these coefficients correspond to the three well known time derivatives used in literature: the contravariant convected time derivative; the covariant convected time derivative and the corotational or Jaumann derivative. Another derivative based exclusively on $\boldsymbol{\Omega}$ called corotational-eigenvectors-of- $\boldsymbol{S}$ derivative, is also obtained.

These possibilities can be put together in Eq. (8) replacing $c_{1}$ and $c_{2}$ with two new coefficients $\alpha_{1}=1-c_{1}$ and $\alpha_{2}=1-c_{2}$ :

$$
\begin{array}{r}
-\frac{\Gamma}{I_{\tau}}\left(\frac{D I_{\tau}}{D t}+\frac{I_{\tau}}{D e}\right)+\left(\alpha_{1}(S \Gamma+\Gamma S)-\frac{2}{3}\{\Gamma S\} I\right)- \\
-\alpha_{2}(\bar{W} \Gamma-\Gamma \bar{W})+2\left(\frac{\eta_{1}}{D e}+\frac{1}{3} I_{\tau}\right) S=0,
\end{array}
$$

where $\bar{W}=W-\Omega$, and the four particular cases can be summarized as

i) $\alpha_{1}=\alpha_{2}=0$, contravariant convected time derivate,

ii) $\alpha_{1}=2$ and $\alpha_{2}=0$, covariant convected time derivate,

iii) $\alpha_{1}=1$ and $\alpha_{2}=0$, corotatinal derivate,

iv) $\alpha_{1}=\alpha_{2}=1$, corotational-eigenvectors-of-S derivate

With this approximation it is now possible to transform Eq. (8) into an algebraic tensor equation to be solved explicitly $\left(\Gamma=\Gamma\left(S, \bar{W}, I_{\tau}\right)\right)$. The expression for the explicit algebraic extra-stress is obtained using a general polynomial tensor for the kinematic tensor, for the representation of $\boldsymbol{\Gamma}$. In the frame of Newtonian turbulent flows, Pope, 1975 has used a tensor polynomial expansion to obtain an explicit algebraic representation for the Reynolds stress tensor equation. With an analogous procedure, Gatski and Speziale 1993 extended this analysis to three dimensional flows obtaining an algebraic stress model from a differential second-moment closure for turbulent flows. Since the tensor $\boldsymbol{\Gamma}$ is symmetric and traceless, the same ideas are applied here and this tensor can be represented as:

$$
\Gamma=\sum_{n=1}^{N} \beta_{n} T^{(n)}
$$

where the basis $T^{(n)}$ is given symmetric traceless tensor functions of $S$ and $\bar{W}$ and the scalar coefficients $\beta_{n}$ functions of the invariants of $\Gamma, S$ and $\bar{W}$.

Using the Eq. (9) for the trace $I_{\tau}$ and expressing Eq. (13) with the three-term base: $T^{(1)}=S, T^{(2)}=S \bar{W}-\bar{W} S$ and $S^{2}-\frac{1}{3}\left\{S^{2}\right\} I$., that gives an exact representation for 2-D flows, we have:

$$
\Gamma=\frac{1}{\left\{S^{2}\right\}}\left[\left(\frac{\eta_{1}}{D e}\right)+\left(\frac{\left(\alpha_{1}\right)^{2}}{2}-\frac{\alpha_{1}}{3}+\frac{1}{3} I_{\tau}\right) I_{\tau}\left\{S^{2}\right\}+\frac{\left(\alpha_{2}\right)^{2}}{2} I_{\tau}^{2}\left\{\overline{W^{2}}\right\}\right]^{1 / 2} S+
$$




$$
\frac{I_{\tau}}{\left\{S^{2}\right\}}\left[\frac{\alpha_{2}}{2}(S \bar{W}-\bar{W} S)+\left(3 \alpha_{1}-2\right)\left(S^{2}-\frac{1}{3}\left\{S^{2}\right\} I\right)\right] .
$$

The use of the general derivative Eq. (11) and the new formulation with the coefficients $\alpha_{1}$ and $\alpha_{2}$ have given a flexibility to the model as it will be shown on the next section.

\section{Predictions for $\mathrm{N}_{2}$}

The Oldroyd-B constitutive equation (c. e.) can not predict the second normal stress difference in shear, $N_{2}$. In fact, using a dimensional approach, it can be shown that, in shear, the predictions for the tensor $\boldsymbol{\Gamma}$ with the Oldroyd-B constitutive model are the following:

$$
[\Gamma]=\left[\begin{array}{ccc}
\frac{2}{3} N_{1} & 0 & 2_{1}^{*} S_{13} \\
0 & -\frac{1}{3} N_{1} & 0 \\
2 \eta_{1}^{*} S_{13} & 0 & -\frac{1}{3} N_{1}
\end{array}\right]
$$

where $\$ N_{-}\{1\}$ is the first normal stress difference in pure shear flow. While for the AESM ( $\backslash$ ref \{eq: aesmgamma \}), the predictions, in shear, for $\$ \backslash \mathrm{mbox}$ \{\boldmath\$ $\backslash$ Gamma $\$$ \} are:

$$
[\Gamma]=\left[\begin{array}{ccc}
\frac{1}{2} N_{1}\left(\alpha_{1}+\alpha_{2}-\frac{2}{3}\right) & 0 & 2_{1}^{*} S_{13} \\
0 & \frac{1}{2} N_{1}\left(\alpha_{1}-\alpha_{2}-\frac{2}{3}\right) & 0 \\
2 \eta_{1}^{*} S_{13} & 0 & N_{1}\left(-\alpha_{1}+\frac{2}{3}\right)
\end{array}\right]
$$

If one wants to maintain the same prediction as the Oldroyd-B model for shear with AESM, a comparison between Eq. (14) and Eq. (15) would lead to a conclusion that $\alpha_{1}=\alpha_{2}=1$ are the proper choices. It is easy to show that for a real shear flow the traceless tensor $\boldsymbol{\Gamma}$ is given by:

$$
[\Gamma]=\left[\begin{array}{ccc}
\frac{1}{3} N_{1}\left(2 N_{1}+N_{2}\right) & 0 & 2{ }_{1}^{*} S_{13} \\
0 & -\frac{1}{3}\left(N_{1}-N_{2}\right) & 0 \\
2 \eta_{1}^{*} S_{13} & 0 & -\frac{1}{3}\left(N_{1}+2 N_{2}\right)
\end{array}\right]
$$

When Eq. (15) and Eq. (16) are compared, it can be seen that

$$
\alpha_{1}=1+\frac{2}{3} \frac{N_{2}}{N_{1}}
$$

and $\alpha_{2}=1$, would give AESM a prediction for $N_{2}$. If the material has no second stress difference, the same previous values for the coefficients are found, namely, $\alpha_{1}=\alpha_{2}=1$. Therefore, even in this case, where AESM model is originated from the Oldroyd-B c. e., the capability of prediction for the components of the traceless stress tensor of the real shear flow has increased with this formulation. A more general conclusion is that depending on the choice of the parameters $\alpha_{1}$ and $\alpha_{2}$, a certain rheological function is predicted more or less precisely. This conclusions gives an important reason for an investigation of which kind of kinematics the material is going through. For example, if the flow is predominantly extensional, then, other guidelines (good prediction of extensional rheological properties) are given for proper choices of the parameters $\alpha_{1}$ and $\alpha_{2}$.

\section{Flow-type sensitive classifier}

In order to characterize the flow, the criterium presented in Astarita, 1979, based on the invariance of the relative-rateof-rotation tensor, is used here. The deformation rate parameter $\left(s^{2}\right)$ and the rotation rate parameter $\left(w^{2}\right)$ are defined respectively as:

$$
s^{2}=\operatorname{tr}\left(S^{2}\right)=S_{m n} S_{n m} \text { and } w^{2}=-\operatorname{tr}\left(\bar{W}^{2}\right)=-\bar{W}_{m n} \bar{W}_{n m} .
$$

Defining $R$ as the ratio between these two quantities:

$$
R=\frac{w^{2}}{s^{2}}=-\frac{\bar{W}_{m n} \bar{W}_{n m}}{S_{m n} S_{n m}}
$$

which will take values from 0 to $\infty$. This kind of parameter can be used to classify flows. It is local, objective, is not restricted to a class of flow and is entirely kinematic (there is no material property involved). It is a measure of how much the material avoids stretching through a rotation (related to the principal directions of stretching). In this sense, it is expected that at one side of the limiting cases stands the extensional kinematics, because the material is not avoiding to be stretched at all; while at the other side is the rigid body motion, when the material is not stretched. In fact, it can be shown (Astarita, 1979) that for pure extensional flow, $R=0$ and as the flow approximates to a solid body motion, $R \rightarrow \infty$. Every other kind of flow lies in between, including pure shear flows, where $R=1$, which is considered to be another limiting case (on a 2-D Cartesian flow the value $R=1$ is the supremum for elliptical flows (Astarita, 1991), and a infimum for strong flows, (Tanner and Huilgol, 1975). This parameter was used in constitutive models first in Schunk and Scriven, 1990, where extensional and shear viscosity effects were decoupled; in

Souza Mendes et al., 1995, where a new weight function for the viscosity was proposed and the prediction for the second normal stress difference was included and in (Thompson et al., 1999), where all the rheological functions for stationary flow, in shear (including the first normal stress difference) and extension are being carried to regions of complex flows.

In the results, this quantity is normalized to avoid numerical problems when $R \rightarrow \infty$ : 


$$
D=\frac{1-R}{1+R}
$$

The scalar $D$ will take values between -1 and +1 . For pure shear flow, $D=0$; for pure extensional flow, $D=1$ and as the flow approximates to a solid body motion, $D \rightarrow-1$. This parameter is used, in this present work, as a guide for the analysis of the comparisons that are made. As this quantity is entirely kinematics, the Newtonian solution is used to produce a $D$ field. With this map that places the type of kinematics that the fluid is going through, there were chosen axial positions which would make physically relevant the comparisons between the cases. Besides that, this parameter was essential on the interpretation of this results.

\section{NUMERICAL METHOD}

In order to obtain the extra-stress components for the Oldroyd-B fluid, the viscoelastic numerical simulations presented in this work are doing using two different set of equations: i) the constitutive equations in a differential form and ii) the algebraic extra-stress model (AESM).

To solve these equations a finite volume numerical method has been successfully employed. The spatial discretisation is performed in a staggered grid (Patankar, 1980). The non-linear terms (convective flux) of the momentum equations, the advection terms of the constitutive equations for the Oldroyd-B differential model, and the advection terms for the trace of the extrastress tensor (when using the AESM formulation), are obtained by a second-order accuracy scheme, namely QUICK (Quadratic Upstream Interpolation Scheme for Convective Kinematics) proposed by Leonard, 1979\}. The diffusion terms of the momentum equations are calculated with the second-order accuracy centered difference scheme. The pressure and the normal stress components of the viscoelastic tensor are treated at the center of the control volumes. The velocities are staggered and evaluated at the center of the faces; the off-diagonal components of the viscoelastic tensor are attached to nodes at the mid-edges. In order to evaluate the shear extra-stress component (off-diagonal term), for example $\left(\left(\tau_{13}(i, k)\right)\right.$, its equation is solved at the center of the cell with the normal components, and then the values are

extrapolated linearly for the corner of the cell. A regular spaced mesh with 3.000 nodes ( 75 nodes in the streamwise direction $(x)$ and 40 nodes in the normal direction $(z)$ has been used. The grid has been built using the following geometrical dimension: $20 \mathrm{H}$ for the streamwise direction and $4 \mathrm{H}$ for the normal direction, where $\mathrm{H}$ is half the height of the outflow channel (see Fig.1) The used regular mesh presents 60 nodes before the entrance $\left(L_{e}=16 h\right)$ and 15 nodes after the entrance $\left(\left(L_{0}=4 H\right)\right)$. In the region after the abrupt entrance, 10 nodes were used $\backslash$ to discretized the outflow channel in the normal direction.

The decoupling procedure employed for the pressure is derived form the work of Harlow and Welch, 1965. Details of this algorithm used to solve viscoelastic flows have been well documented in a previous work (Mompean and Deville, 1997), and will be briefly described here.
From the momentum conservation equation, a discrete Poisson equation is obtained for the pressure by enforcing the mass conservation implicitly. In the momentum equation, the diffusion terms due to the Newtonian stresses (solvent) and due to the extra-stress components (viscoelastic fluid) are treated explicitly. The non-linear terms are also evaluated explicitly. The differential constitutive equation for the Oldroyd-B fluid is solved using an Euler explicit scheme. With such information, the right hand side $(B)$ of the linear system for the pressure is evaluated at time $n$, and the new pressure values are calculated at time $n+1$ :

$$
[A] p^{n+1}=B^{n}
$$

The matrix $[A]$ of the system, used to obtain the pressure (p) at $n+1$, is symmetric and positive definite. It can be solved by a direct Cholesky factorization or by a preconditioned conjugate gradient method. This scheme represents a real evaluation in time (no pseudo-transient algorithm is used here). The steady-state solution is computed by the convergence of a time dependent process.

\section{Boundary conditions}

For the momentum equation the same boundary conditions were used when solving the Oldroyd-B differential constitutive equations or when solving the AESM. These conditions were:

1) At the inlet a parabolic profile is given for the $U$ velocity.

2) At the walls, the no-slip condition is applied to the velocities.

3) At the outlet of the domain a Neumann condition is used for all variables, except the pressure. A zero reference value is given for the pressure at the exit.

When solving the Oldroyd-B constitutive equations, the extra-stress components are deduced from a plane Poiseuille flow and, and at the inlet of the domain the following values are given:

$$
\begin{aligned}
\tau_{11} & =2 \eta_{1} D e(\partial u / \partial z)^{2}, \\
\tau_{33} & =0, \\
\tau_{13} & =\eta_{1}(\partial u / \partial z) .
\end{aligned}
$$

For the AESM, in the inlet only one boundary condition has to be given (the trace of the extra-stress tensor) to the viscoelastic model. For a developed Poiseuille flow the trace is:

$$
I_{\tau}=2 \eta_{1} D e(\partial u / \partial z)^{2}
$$




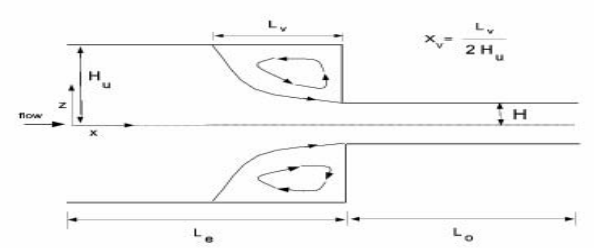

Figure 1: Geometrical parameters for the contraction.

\section{RESULTS}

For a first study of the new theory presented, it was decided to compare only the stress tensor of the different models. So the calculation that was done, was based on a given kinematics. On the present work, the velocity field was the Newtonian solution of the problem.

\section{Newtonian Fluid}

For the numerical simulation of the Newtonian flow through the $4: 1$ contraction, different grids were used in order to check the grid independence in the results. In this section we show the results obtained with a regular spaced grid of $\backslash 3,000$ nodes ( 75 nodes in the streamwise direction $x$ by 40 nodes in the normal direction $z$ ). The influence of the entry upstream length $\mathrm{L}_{\mathrm{e}}$ on the results was verified; to permit the development of the flow in the entry region, the ratio 4 between the entry upstream length $\left(\mathrm{L}_{\mathrm{e}}\right)$ and the outlet downstream length $\left(\mathrm{L}_{0}\right)$ was used (see Fig. 1), which corresponds to corner step at $x=16$. The dimensionless size of the recirculation, $X_{V}=L_{V} / 2 H_{U}$, is equal to 0.18 , which is in good agreement with previous calculation for this flow (Phillips and Williams, 1999).

As a first step, to verify the influence of new terms in the frame-invariant model, the eigenvectors of the strain deformation stress tensor $\boldsymbol{S}$ have been calculated for a Newtonian creeping flow through a contraction. The Reynolds number based on the averaged exit velocity and on the downstream height was 0.01 .

\section{Predictive capability of the AESM}

\section{The flow-type classifier - $D$}

The contour values for parameter $D$ inside the $4: 1$ contraction are shown on Fig. 2. The three limiting types of flow (extensional, shear and solid body) are clearly found.

The regions near the horizontal walls, where the flow is developed close to the inlet and to the outlet, are characterized by pure shear, which corresponds to values of the deformation parameter $D$ going to zero.

Just before the entrance of the abrupt contraction, a region of pure elongational flow is shown, which is represented by the white contours, where the values of $D$ are close to one. Another region where the flow starts to elongate is where the streamlines curve to enter at the smaller section. These two regions can be seen as parts of the same region (because they depend on the range of $D$ choices). It is expected that the presence of a contraction, or equally, the passage to a region where (for incompressible fluids) the mean velocity is higher, cause stretching to the fluid. For a material filament aligned with the velocity vector that presence affects the forward point of this filament strongly then the back the backward and as a consequence an original filament located at the left side of the contraction has a higher length.

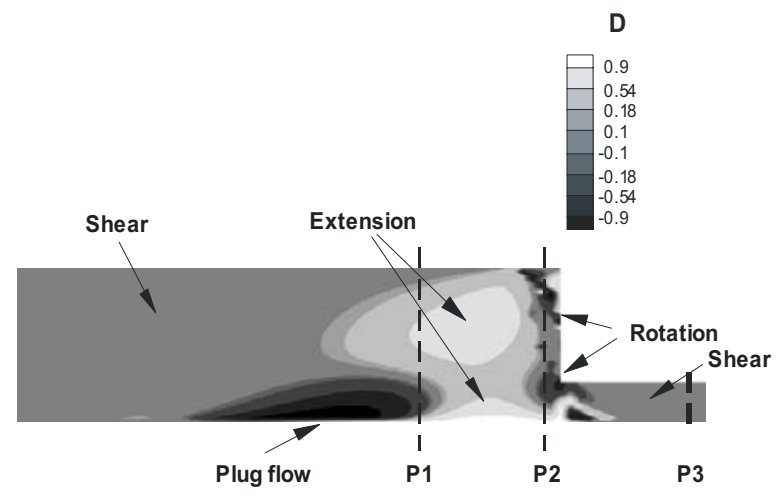

Figure 2. Isobands of the parameter D flow classifier

Concerning the elliptical flows (flows between shear and solid body motion), three regions are presented where the values of the parameter $D$ become negative: a) the first one, just after the sharp corner; b) a second one near the corner vortex, in the vertical wall; and c) the third one just before the extensional region at the entrance of the smaller section. The two first regions are easy to justify: at the corner there are closed vortices that rotate and it is expected that the rotation rate there is to be bigger then the deformation rate; at the lip, the near fluid has to make a hard orthogonal turn and the rate of rotation again is bigger. The third region, can not be seen as rotation motion but as a motion close to a "plug-flow". At a first glance it is strange that between a region of (expected) shear $(D=0)$ and of (expected) extension $(D=1)$ appears a region of rigid body motion $(D \rightarrow-1)$. The explanation here (for details, see Thompson 2001\}) is done by examining what is happening, separately, with the deformation and the rotational rate parameters Eq. (18). The first thing to notice is that the deformation rate at the shear region vanishes at the centerline and has its maximum at the wall. So, concerning a particle that is near to the centerline region, it starts from a low deformation rate, but the eigenvectors of $\boldsymbol{S}$ have to change their direction: from the region of shear, where the principal directions are at $\pm \frac{\pi}{4}$ rotated axis, to the region of extension, where they are aligned with the axis. The results indicate that for this region, changes on the eigenvectors directions are done with higher gradients then the changes on deformation rates. It can be seen that the opposite effect happens for regions far from the centerline where transition from shear to extension is done in an smooth (concerning the $D$ parameter) way. 


\section{Comparison between the particular cases}

The three axial positions marked with a dashed line, namely, P1, P2 and $\mathrm{P} 3$, respectively at dimensionless positions $\mathrm{x}=12, \mathrm{x}=15.5$ and $\mathrm{x}=18$; shown on Fig.2, were chosen for a deeper study at the components of the traceless tensor $\boldsymbol{\Gamma}$. The first one, P1, is a position where there are all kinds of motion along the axe, and the changes are done in a smooth way. Position P2, is where really strong gradients of $D$ occur. It takes the vortex region, the region near the lip and the extensional region close to the centerline. And the third one, P3, is a position where the fluid, along the line, does not go through changes on its type of flow, which is pure shear. The comparisons are made for six particular cases corresponding to: the Oldroyd-B constitutive equation, the

non-objective one, AESM- $W$, and four others objective AESMs (all of this five Oldroyd-B based), each of them corresponding to the particular forms of the general frameindifferent time derivative Eq.(11) .

Position P3, was used to validate the results obtained by the numerical code, as the solution for viscometric flow can be calculated analytically by equations Eq.(14) and Eq.(15). For the $\Gamma_{11}$ AESM objective component, the coefficient multiplying $N_{1}$ is $\frac{1}{2}\left(\alpha_{1}+\alpha_{2}-\frac{2}{3}\right)$. It was seen an exact agreement between the three models that have the same value for $\alpha_{1}+\alpha_{2}$ and the excellent agreement with $\Gamma_{11}$ component obtained with the OldroydB c. e. The other two behaviors were also consistent, including the negative values for the case $\alpha_{1}=0, \alpha_{2}=0$; as in this case, $\alpha_{1}+\alpha_{2}<\frac{2}{3}$. The cross component was also compared. It was seen that in this case the predictions were again confirmed by the analytical results. It is a linear function with the normal axe, as the shear rate.

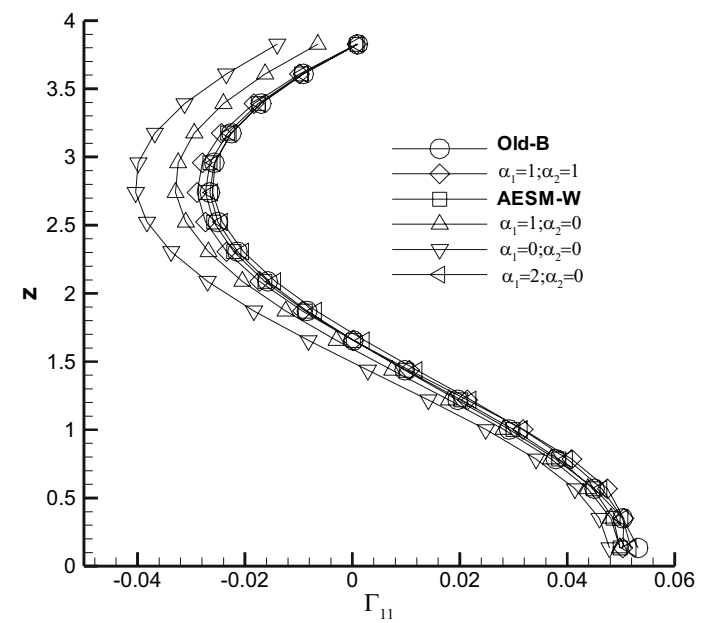

Figure 3. $\Gamma_{11}$ at the position $\mathrm{P} 1(\mathrm{x}=12)$

Figures 3 and 4 are showing the $\Gamma_{11}$ and $\Gamma_{13}$ components, of the traceless tensor, for the six cases at position P1. The qualitative results are the same for all cases. For the $\Gamma_{13}$ component there is no difference for the five cases with the AESM formulation. There is an exact agreement concerning the points close to the wall and very near the centerline. The Oldroyd-B results differ a little at the region corresponding to the transition from the plugflow region to the extensional one.

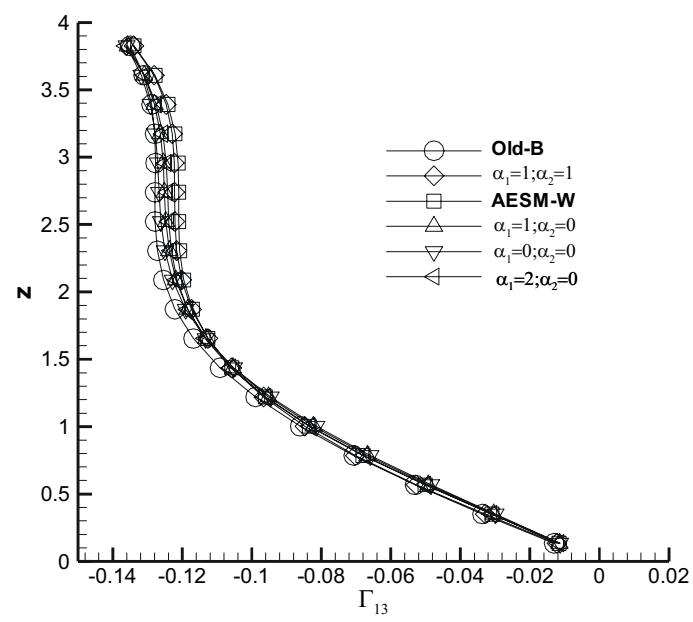

Figure 4. $\Gamma_{13}$ at the position $\mathrm{P} 1(\mathrm{x}=12)$

This difference can be caused by the numerical code. For the $\Gamma_{11}$ component it can be seen that all the cases are bounded by the contravariant $\left(\alpha_{1}=0, \alpha_{2}=0\right)$ case from the left and the covariant $\left(\alpha_{1}=2, \alpha_{2}=0\right)$ from the right. Generally, the cases where $\alpha_{1}+\alpha_{2}=2$ have a similar behavior to the one obtained with the Oldroyd-B c. e. They present a more similar behavior to each other near the centerline.

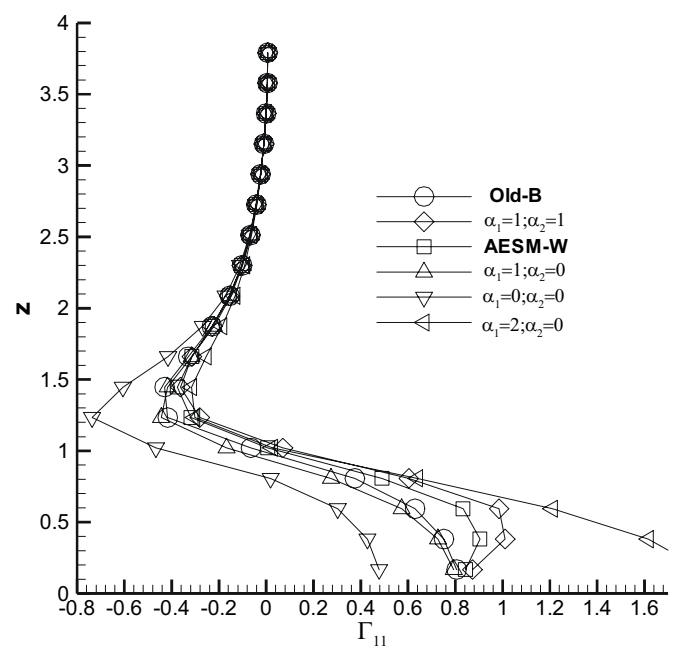

Figure 5. $\Gamma_{11}$ at the position $\mathrm{P} 2(\mathrm{x}=15.5)$

At position $\mathrm{P} 2$, the results obtained for the cross component $\Gamma_{13}$ were analogous to the ones at position P1, in the sense that all the cases with the AESM formulation were coincident. 


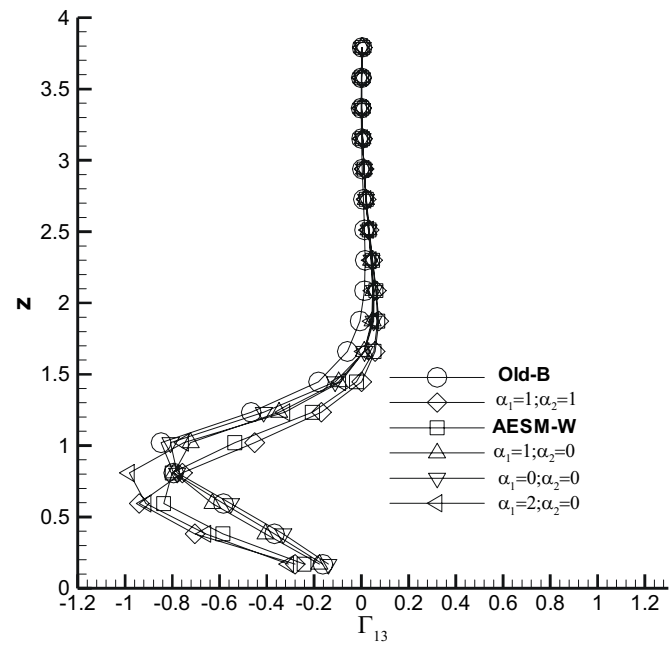

Figure 6. $\Gamma_{13}$ at the position $\mathrm{P} 2(\mathrm{x}=15.5)$

Fig. 5 shows, also, the good agreement with ones obtained with the original differential constitutive equation, including the region of negative values, until $z \approx 1.7$. It can be seen that passing this point, where, as it is shown on Fig. 2, the influence of the singular point (at the entrance of the contraction) is not relevant, $\Gamma_{13}$ vanishes. Concerning predictions for the $\Gamma_{11}$ component, analysis of Fig. 6 shows that is $\alpha_{1}$ (and not $\alpha_{1}+\alpha_{2}$ ) the parameter that governs its behavior. The explanation is that, near the extensional region, $\bar{W}$ is small, and so $\alpha_{2}$, Eq. (8), has no influence.

\section{The second normal stress difference in shear $-N_{2}$}

The other result of interest is the case with a prediction for a non vanishing second normal stress difference in pure shear, $N_{2}$. It is known that the Oldroyd-B c. e. predicts a constant first normal stress coefficient in pure shear, $\Psi_{1}$. Generally, the Deborah number is related to a characteristic time, $\lambda$ based on $\Psi_{10}$, the limit value for $\Psi_{1}$ when $\gamma \rightarrow 0$. As the analytical results has shown Eq. (17), it is necessary to evaluate the ratio $\frac{N_{2}}{N_{1}}$ in order to

find $\alpha_{1}$. An analysis of the rod-climbing phenomena (present only in elastic fluids) with an approximation of a second order fluid was made in Joseph, 1990. This analysis has given the following range for the coefficient,

$\Psi_{20}$ (the limit value for the second normal stress coefficient in pure shear, $\Psi_{2}$, when $\gamma \rightarrow 0$ ) related to $\Psi_{10}:-0.25 \Psi_{10} \leq \Psi_{20} \leq 0$. For many polymeric liquids $\Psi_{20}=-0.1 \Psi_{10}$. So this was chosen as a particular case: $N_{2}=-0.1 N_{1}$. With that assumption, the values for the coefficients of Eq. (8) are $\alpha_{1}=0.9333 \ldots$ and $\alpha_{2}=1$. Obviously, the function $N_{2}(\dot{\gamma})$ is parabolic, as $\Psi_{2}$ is constant, $\dot{\gamma}$ is linear and $N_{2}=\Psi_{2} \dot{\gamma}^{2}$.

\section{CONCLUDING REMARKS}

In the present work was presented a general procedure for obtaining an objective algebraic model for the extra stress tensor (AESM), starting from any differential constitutive model, for a 3-D, transient flow. The process is done towards a decrease on the computation efforts. It reduces the set of differential equations for the components of the extra stress tensor to only one differential equation for its trace and an explicit equation for the traceless stress tensor. This is done by an assumption based on an analogy with turbulent flows in which the extra-stress anisotropy tensor reaches equilibrium, in other words its time derivative vanishes. A general objective time derivative is used to accomplish that assumption and to give flexibility to the model.

These general ideas are applied to the Oldroyd-B constitutive model with a three-term base of tensors (that gives an exact representation for the traceless tensor in 2-D flows) to study the particular case of a steady, 2-D planar, 4:1 abrupt contraction. A theoretical analysis showed the possibility of including a prediction for the viscometric second normal stress difference (that is not predicted by the original model) and indicate that regions where the advection of the stress tensor can be neglected, the Deborah number is limited. The Newtonian velocity field solution of the problem is used to compute and compare the stress tensor for other (then the Oldroyd-B) five different cases of AESM formulation, namely, a nonobjective one (AESM- $W$ ) and four other ones originated by particular choices of the general objective time derivative corresponding to: the contravariant and covariant convected time derivatives, the corotational (or

Jaumman) derivative and to another kind of corotational derivative based on the rate of rotation of the eigenvectors of the deformation rate tensor (AESM- $\bar{W}$ ). A field of a parameter, $D$, that is sensitive to the type of the flow (viscometric, extensional, solid body motion) was used to indicate the positions of comparison and to understand similar and different behaviors between the models. In this way, parameter $D$ was an excellent tool, and can be used not only when calculations are done with the AESM, but in general when different constitutive models are being compared.

For the position of the viscometric flow, the results were the same as predicted by the theoretical analysis: the same cross component of the traceless tensor and the principal normal component dependent on the sum of the two free coefficients of the explicit equation, $\alpha_{1}$ and $\alpha_{2}$. For the other two positions, in general, the results were bounded by the contravariant and covariant time derivatives, but qualitatively, all the cases had similar behavior and quantitatively, it could be seen that AESM$\bar{W}$ and AESM- $W$, sometimes with the covariant derivative and others with the corotational one, could capture the same predictions of the Oldroyd-B model. This has shown that, at least for the studied cases, the basic assumption for the equilibrium of the extra-stress anisotropy tensor was a good approximation and has lead to well behaved solutions. For a general conclusion, the next step is to obtain solutions for the present problem with the cases presented, solving the velocity field, for a range of $R e$ and De.

The inclusion of the prediction for $N_{2}$, was an interesting result. Although the formulation is based on an original differential model, being a simplified version of it (in the sense described on the first paragraph), there was an 
increase in the capability of prediction in a sense that another rheological property is captured. This result indicates that the AESM based on the White-Metzner constitutive model (a generalization of the Oldroyd-B one) that gives a dependence on the shear rate for the shear viscosity, $\eta=\eta(\dot{\gamma})$, and first normal stress coefficient, $\Psi_{1}=\Psi_{1}(\dot{\gamma})$, but does not predict second normal stress coefficient would probably include a prediction, for this rheological function, of the type $\Psi_{2}=\Psi_{2}(\dot{\gamma})$.

Other constitutive models that have a better prediction for the extensional rheological functions can be used to derive more general AESMs . If the parameters $\alpha_{1}$ and $\alpha_{2}$ are generalized to be functions of the second invariants of $\boldsymbol{S}$ and $\bar{W}$ this will allow predictions not only for the limiting cases (shear flow, extensional flow and solid body motion) but to the regions of complex flows to where this information can be carried to.

\section{ACKNOWLEDGMENTS}

This work was funded by grants from the project between CAPES and COFECUB and from FAPERJ.

\section{REFERENCES}

Astarita, G., 1979, Objective and generally applicable criteria for flow classification, "J. Non-Newtonian Fluid Mech.”, Vol. 6, pp. 66-76.

Astarita, G., 1991, Quasi- Newtonian constitutive equations exhibiting flow-type sensitivity, “J. Rheol.", Vol. 35(4), pp.687-689.

Baaijens, F. T. P., 1993, Numerical analysis of start-up planar and axisymmetric contraction flows using multi-mode differential constitutive models, "J. Non-Newtonian Fluid Mech.”, Vol. 48, pp. 147-180.

Binding, D. M., 1988a, An approximate analysis for contraction and converging flows, "Journal of NonNewtonian Fluid Mechanics”, Vol. 27, pp. 173-189.

Binding, D. M., 1988b, Further considerations of axisymmetric contraction flows, "Journal of Non-Newtonian Fluid Mechanics", Vol. 41, pp.27-42.

Binding, D. M., and Walters, K., 1988, On the use of flow through a contraction in estimating the extensional viscosity of mobile plymer solutions, "Journal of Non-Newtonian Fluid Mechanics", Vol. 30, pp.233-250.

Cogswell, F. N., 1972, "Polym. Eng. Sco.”, Vol. 12, pp.64-73.

Evans, R. E. and Walters, K., 1986, Flow characteristics associated with abrupt changes in geometry in the case of highly elastic liquids, "J. Non-Newtonian Fluid Mech.", Vol. 20, pp. 11-29.

Evans, R. E. and Walters, K., 1989, Further remarks on the lip-vortex mechanism of vortex enhancement in planarcontraction flows, “J. Non-Newtonian Fluid Mech.", Vol. 32, pp.95-105.

Gatski, T. B., and Speziale, C. G., 1993, On explicit algebraic stress models for complex turbulent flows, "J. Fluid Mech.", Vol. 254, pp 59-78.

Harlow, F. H. and Welch, J. E., 1965, Numerical calculation of time-dependent viscous incompressible flow of fluid with free surface, "Phys. Fluids", Vol. 8, pp.2182-2189.
Joseph, D. D., 1990, "Fluid Dynamics of Viscoelastic Liquids", Applied Mathematical Sciences, Springer-Verlag, New-York.

Keunings, R., 1986, On the high Weissenberg number problem, “J. Non-Newtonian Fluid Mech.”, Vol. 20, pp. 209-226.

Leonard, B. P., 1979, A stable accurate convetive modeling procedure based on quadratic upstream interpolation, "Comput. Meth. Appl. Mech. Eng.", Vol. 19, pp. 59-98.

Mackay, M. E. and Astarita, G., 1997, Analysis of entry flow to determine elongational flow properties, "J. NonNewtonian Fluid Mech.”, Vol. 70, pp. 219-235.

Mompean, G., Thompson, R. L. and Souza Mendes, P. R., 2003, "A general transformation procedure for differential viscoelastic models", Journal of Non-Newtonian Fluid Mechanics (in press).

Mompean, G., 2001, On predicting abrupt contraction flows with differential and algebraic viscoelastic models, "Computer and Fluids (in press)".

Mompean, G. and Deville, M., 1997, Unsteady finite volume simulation of Oldroyd-B fluid through threedimensional planar contraction, "Journal of Non-Newtonian Fluid Mechanics”, Vol. 72, pp.253-279.

Mompean, G., Jongen, T., Gatski, T., and M., D., 1998, On algebraic extra-stress models for the simulation of viscoelastic flows, "Journal of Non-Newtonian Fluid Mechanics", Vol. 79, pp. 261-281.

Patankar, V. S., 1980, "Numerical heat transfer and fluid flow", Hemisphere Publishing Corporation, New York.

Phillips, T. N. and Williams, A. J., 1999, Viscoelastic flow through a planar contraction using a semi-Lagrangian finite volume method, "J. Non-Newtonian Fluid Mech.”, Vol. 87, pp.215-246.

Pope, S., 1975, A more general effective-viscosity hypothesis, “J. Fluid Mech.”, Vol. 72, pp. 331-340.

Purnode, B. and Crochet, M. J., 1996, Floes of polymer solutions through contractions. Part 1: flows polyacrylamid solutions through planar contractions, "J. Non-Newtonian Fluid Mech.", Vol. 65, pp. 269-289.

Rivlin, R. S., 1957, The relation between the flow of nonNewtonian fluids and turbulent Newtonian fluids, "Q. Appl. Maths", Vol. 15, pp. 212-215.

Rivlin, R. S. and Ericksen, J. L., 1955, Stress-deformation relations for isotropic materials, Ärch. Rat. Mech. Ana", Vol 4, pp. 323-425.

Schunck, P. R. and Scriven, L. E., 1990, Constitutive equation for modeling mixed extension and shear in plymer solution processing, "Journal of Rheology", Vol. 34(7), pp. 1084-1117.

Souza Mendes, P. R., Padmanabhan, M., Scriven, L. E., and Macosko, C. W., 1995, Inelastic constitutive equations for complex flows, "Rheologica Acta", Vol. 34, pp. 209-214.

Tanner, R. I. and Huilgol, R. R., 1975, On the classification scheme for flow fields, "Rheol. Acta", Vol. 14, pp.959.

Thompson, R. L., 2001, "Performance of a new constitutive equation for non-Newtonian liquids (in Portuguese)", PhD thesis, Pontifícia Universidade Católica.

Thompson, R. L., Souza Mendes, P. R., and Naccache, M. F., 1999, A new constitutive equation and its performance in contraction flows, "J. Non-Newtonian Fluid Mech.", Vol. 86, pp. 375-388. 